Thorax (1969), 24, 441.

\title{
Localized amyloidosis of the lower respiratory tract
}

\author{
A. R. MAINWARING, G. WILLIAMS, E. O. W. KNIGHT, \\ A N D H. F. M. B ASSETT
}

From the Departments of Pathology and Surgery, Manchester Royal Infirmary

The clinical and pathological findings in a case of amyloidosis localized to the lower respiratory tract are described. We think this is the first report of a female patient with multiple deposits limited to the bronchial tree. The vascular areas in some amyloid deposits were considered to be secondary to pre-existing infection. The fine structure of the amyloid conforms to that of amyloid at other sites in man and in experimental animals.

Amyloidosis localized to the lower respiratory tract is uncommon. Prowse (1958) reviewed 16 cases from the literature and added two more of his own. This paper describes an additional case where the diagnosis was made during life and in which a detailed study of the lungs and bronchial tree was later carried out at necropsy.

\section{CASE REPORT}

A 49-year-old housewife was first admitted to the Manchester Royal Infirmary in January 1967 complaining of severe wheezing and increasing breathlessness. Her respiratory symptoms had begun in 1963 and during the next four years she had repeated chest infections and haemoptysis on several occasions. Sputum cultures repeatedly grew Haemophilus influenzae, but despite various therapeutic measures there was no great improvement in her respiratory condition. A chest radiograph in 1965 had shown no abnormality.

On admission she was slightly cyanosed and had inspiratory difficulty with stridor: there was diminished expansion on the left side of the chest with poor air entry and an inspiratory wheeze. The chest radiograph showed increased radiolucency of the left lung with mediastinal displacement to the right, and so a diagnosis of partial obstruction of the left main bronchus was made.

BRONCHOSCOPY FINDINGS The proximal $1.5 \mathrm{~cm}$. of the left main bronchus was almost completely occluded by a pale gelatinous mass intimately attached to its entire circumference. This mass was removed piecemeal. The vocal cords, trachea, right main bronchus, and lobar bronchi were normal.
FIG. 1. Wall of left main bronchus thickened by amyloid. The lumen is stenosed.

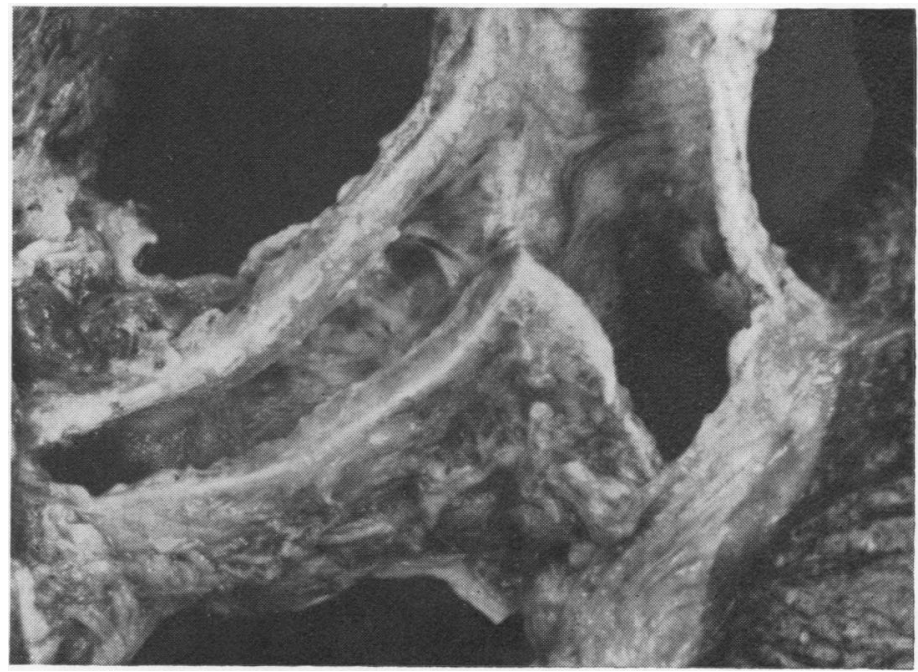


MICROSCOPY Microscopy of the bronchial fragments showed that the bronchial submucosa was infiltrated by an amorphous deposit which gave positive staining reactions for amyloid.

Following bronchoscopy the patient's breathing greatly improved and the chest radiograph returned to normal.

She remained well until May 1968, when she was readmitted because of rapidly increasing breathlessness and a further haemoptysis. She was again slightly cyanosed and had inspiratory stridor and diminished expansion of both sides of the chest. Her chest film again showed over-inflation of the left lung and collapse of the right lower lobe.

BRONCHOSCOPY Bronchoscopy showed stenosis of the left main bronchus at the site of the mass previously removed. The mucosa of the right main bronchus was congested and its lumen almost completely occluded. The thickened mucosa was cored out, which led to profuse bleeding controlled by $1: 1,000$ adrenaline-soaked pledgets.

She was maintained on artificial ventilation through a tracheostomy, but continued to bleed into the lungs and died on the fifth post-operative day.

NECROPSY The trachea was inflamed and partly ulcerated. The upper and lower lobes of the right

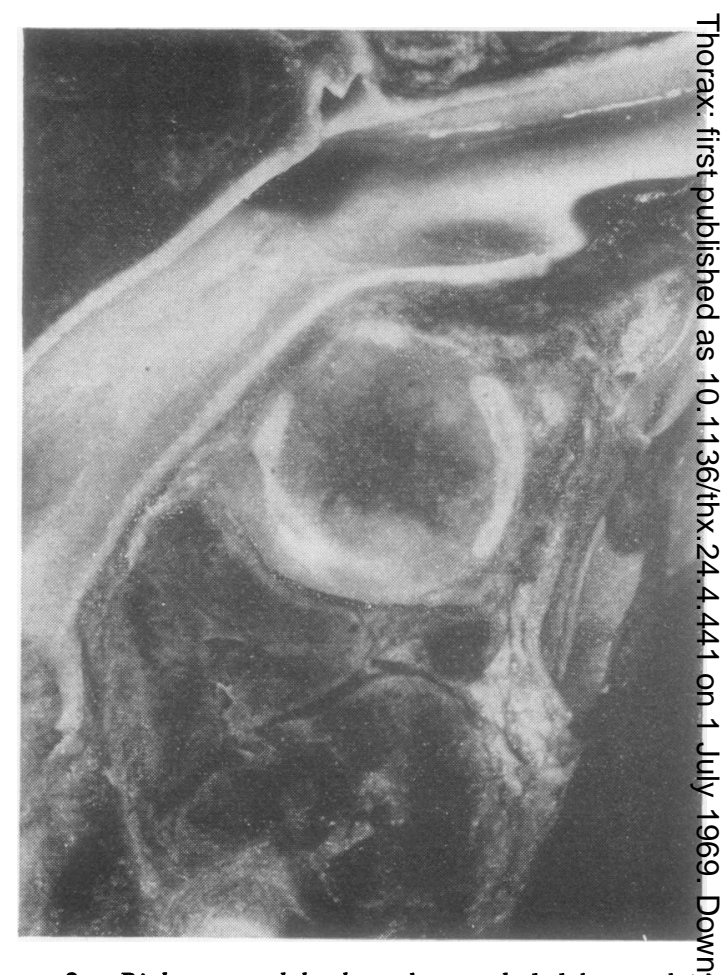

FIG. 2. Right upper lobe bronchus occluded by amyloiळ having a grey, glassy appearance.

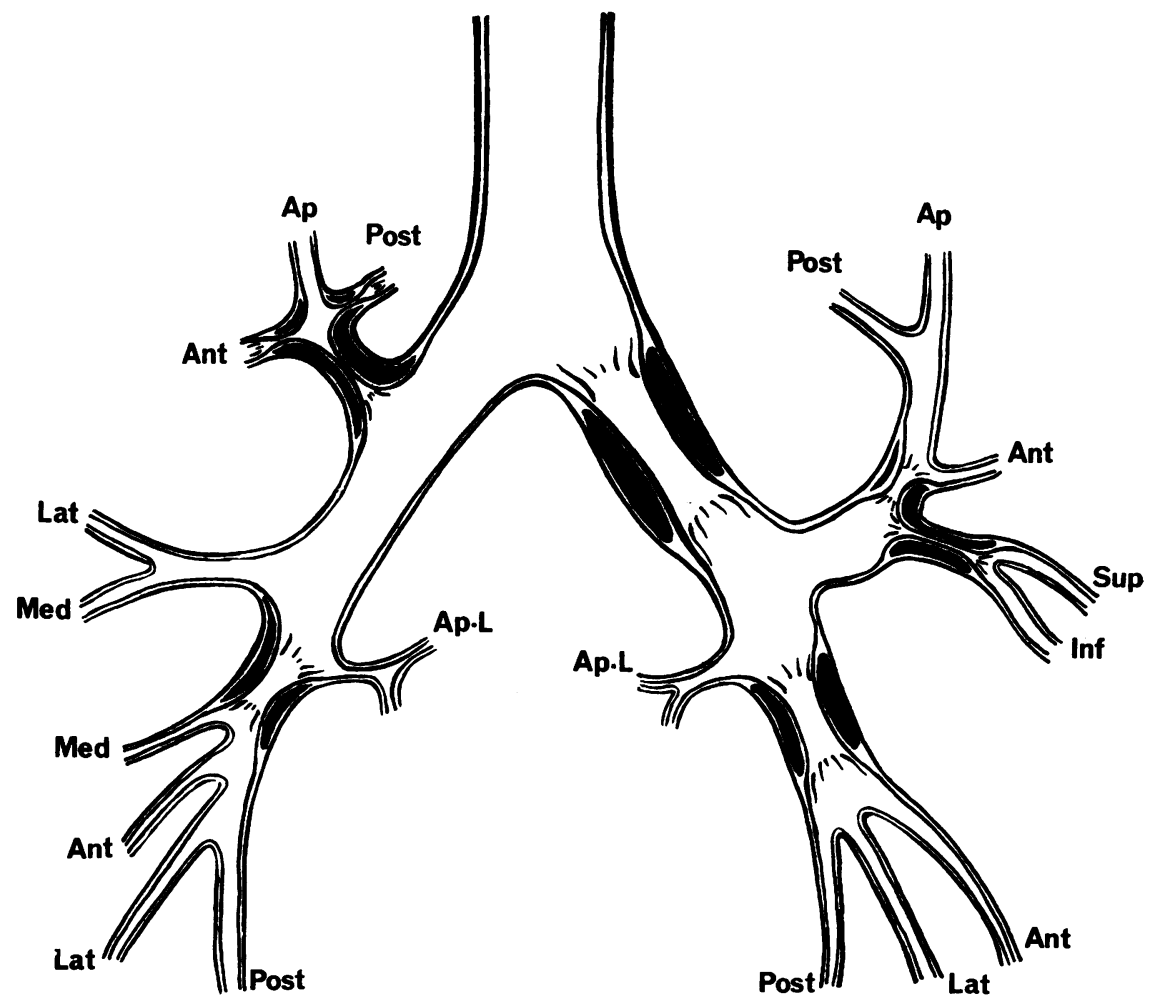

FIG. 3. Sites of amyloid deposits in the bronchial tre?. 
lung were collapsed. The left lung was overdistended and showed bronchopneumonia in its lower lobe. The bronchi were generally inflamed and the right lower lobe bronchus was totally occluded by blood clot. Dissection of the bronchial tree showed a pale orange thickening of the whole circumference of the left main bronchus for a distance of $2.5 \mathrm{~cm}$. (Fig. 1). The entire length of the right upper lobe bronchus was occluded by grey, glassy material (Fig. 2). This thickening extended into the anterior and posterior segmental bronchi. Similar localized thickenings were found in the main right and left lower lobe bronchi, in the lingular bronchus, and in the main left upper lobe bronchus, as indicated in Figure 3.

HISTOLOGY OF BRONCHIAL LESIONS The submucosa was infiltrated by a structureless eosinophilic material which reacted metachromatically to crystal violet and showed a variable affinity for Congo Red which gave a dichroic effect under polarized light. Thioflavine $T$ gave a greenish fluorescence with ultraviolet light. The material thus reacted as classical amyloid and was arranged in strands around normal or atrophic mucous glands and infiltrated by scanty lymphocytes and plasma cells (Fig. 4). The overlying mucosa was partly ulcerated ; intact areas were covered by normal columnar epithelium. In some areas the amyloid surrounded the bronchial cartilage and extended into the alveolar septae. The amyloid was for the most part avascular, but occasionally it surrounded numerous dilated capillary vessels (Fig. 5). There were no giant cells or areas of ossification in any of the lesions.

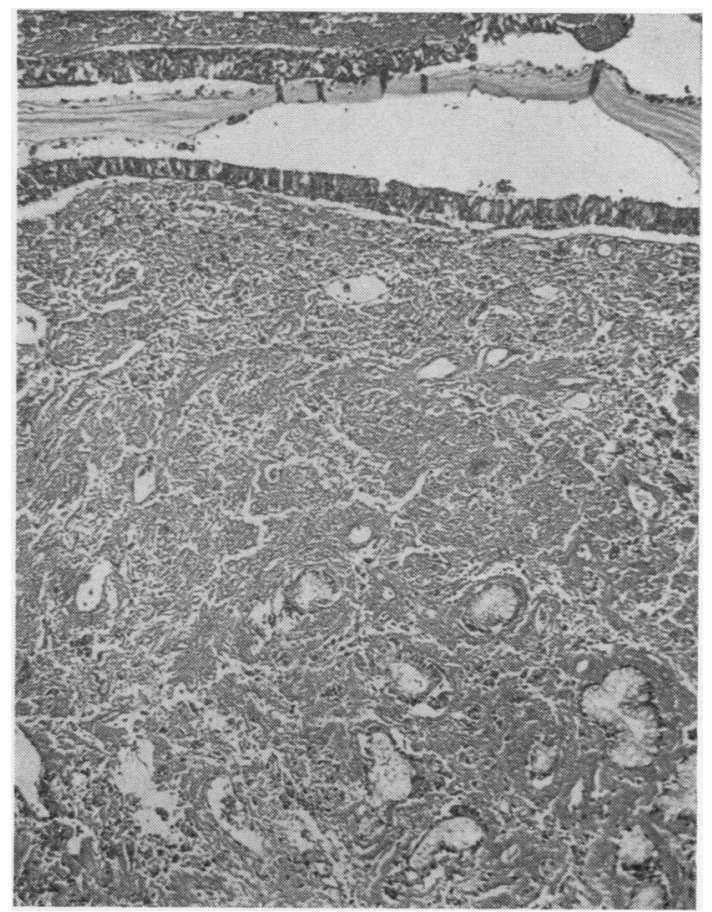

FIG. 4. Amyloid surrounding mucous glands and extending up to the intact epithelial surface. Note scanty inflammatory cells and avascularity (Haematoxylin and eosin $\times 65$ ).
FIG. 5. Numerous dilated vascular channels surrounded by amyloid (Haematoxylin and eosin $\times 30$ ).

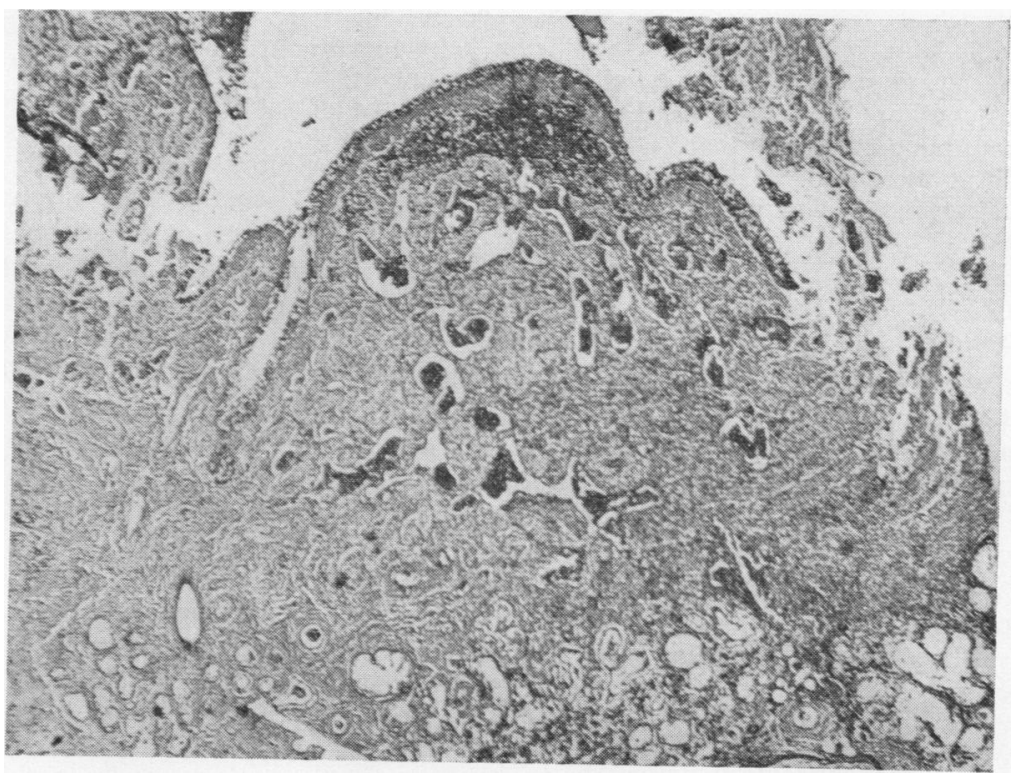




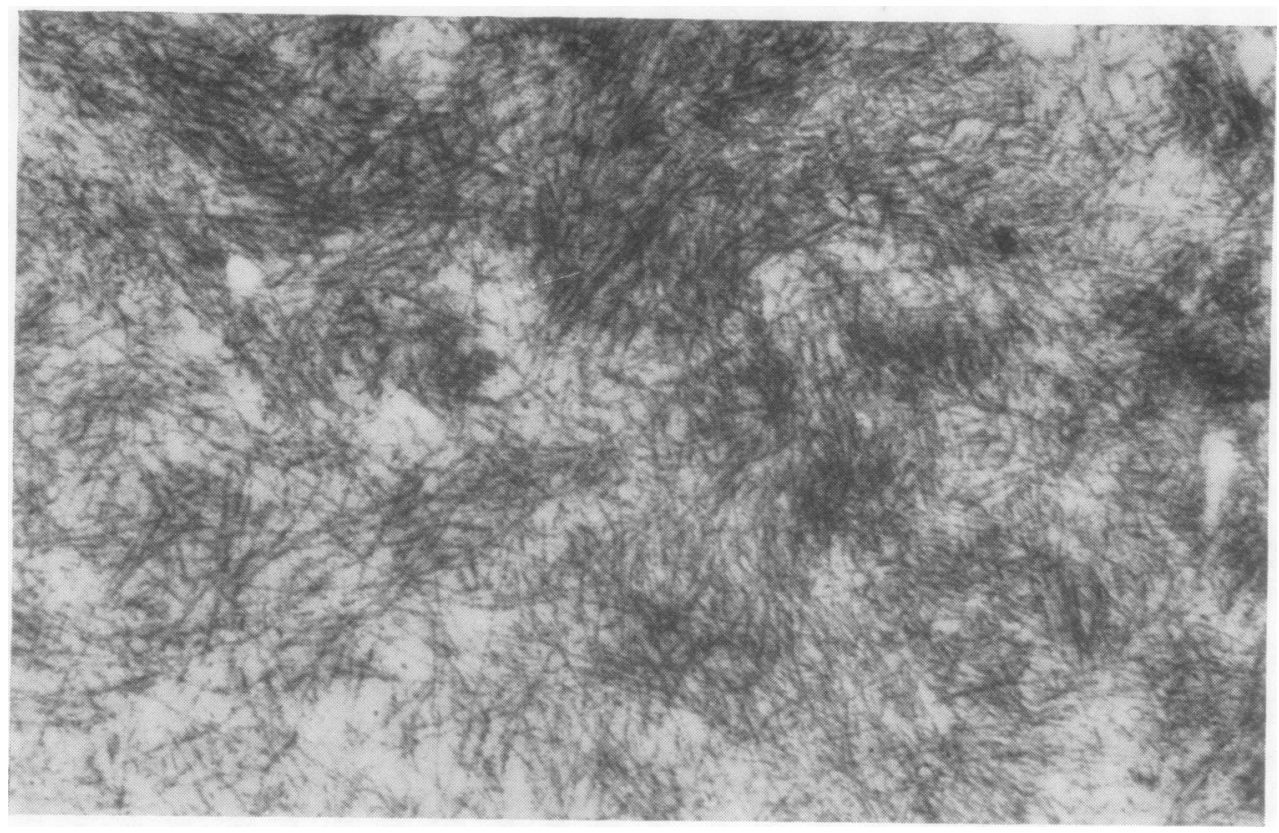

FIG. 6. Amyloid infiltrate showing fibrillar and amorphous components $(\times 30,000)$.

No amyloid deposits were found in any other organs. Histological sections of the sternal and vertebral bone marrow showed no increase of plasma cells.

ELECTRON MICROSCOPY Tissues from the various bronchial lesions were examined. The bronchial walls were infiltrated by amyloid lying between collagen and smooth muscle bundles and in some areas around capillary networks. The amyloid consisted of amorphous and fibrillar components (Fig. 6), the fibrils measuring 80-100 $\AA$ in diameter and being of indeterminate length.

\section{DISCUSSION}

Amyloidosis has been classified into (1) primary amyloidosis, which may occur in a systemic or localized form, (2) generalized secondary amyloidosis, and (3) amyloidosis associated with multiple myeloma (Symmers, 1956).

Prowse (1958) has emphasized the importance of subdividing amyloidosis of the lower respiratory tract into three groups: (1) multiple amyloid deposits confined to the lung parenchyma ; (2) a solitary mass of amyloid affecting a major bronchus; and (3) amyloid deposits involving the wall of many of the air passages (diffuse tracheo-bronchial amyloidosis).
The present case falls into group 3 of Prowse's classification and we think it is the first femalecase to be described where distribution has beeno limited to the bronchial tree. The other two female cases with multiple bronchial deposits have alsoo shown tracheal involvement (Prowse, 1958).

In group 3 there has been a constant clinicalopattern related to the obstructive nature of the 3 . disease. There has always been a history, of variable length, of recurrent chest infections, wheezing, stridor, and progressive respiratory distresso encountered singly or in combination. In the pre sent case it appears that amyloid has probablyo been in the bronchial tree for about five years.

Haemoptysis occurred in this and several othero recorded cases (Whitwell, 1953 ; Gordon, 1955) N 0 Prowse (1958) related the bleeding to haemor-N rhage from infected bronchial mucosa betweeno the amyloid deposits which he considered to be avascular. Fors and Rydén (1964) viewed their angiomatoid amyloid lesion as possibly a vascular tumour or hamartoma. In our case the occasionait vascularized amyloid foci probably arose in pre-0 existing inflammatory granulation tissue whose vessels later became enveloped by amyloid. $\overrightarrow{\mathbb{D}}$

Cases in group 3 have the poorest prognosis, as there are multiple sites of bronchial narrowing to produce infection or pulmonary collapse. Piece 
meal removal of the amyloid is the only way of relieving bronchial obstruction and prolonging life (Schmidt, McDonald, and Clagett, 1953).

On account of their 'atypical' staining reactions, localized amyloid deposits have been classified as 'para-amyloid', inferring a chemical difference from 'typical' or secondary amyloid. The deposits in our case and in others (Cotton and Jackson, 1964) reacted positively with classical amyloid stains, and furthermore the fine structure conforms to that of amyloid described in other sites in man (Spiro, 1959) and in experimental animals (Sorenson and Shimamara, 1964). Its mode of origin is unknown.

We are indebted to Mr. D. Summerfield, H.M. Coroner, and Dr. J. B. L. Howell for permission to publish the case report. We thank Mr. G. C. W.
Humberstone and the Department of Medical Art, University of Manchester, for the illustrations.

\section{REFERENCES}

Cotton, R. E., and Jackson, J. W. (1964). Localised amyloid 'tumours' of the lung simulating malignant neoplasms. Thorax, 19, 97.

Fors, B., and Rydén, L. (1964). Tumoral amyloidosis of the lung. Acta path. microbiol. scand., 61, 1.

Gordon, W. (1955). Amyloid deposits in the bronchi. Brit. med. J., 1,825 .

Prowse, C. B. (1958). Amyloidosis of the lower respiratory tract. Thorax, 13, 308.

Schmidt, H. W., McDonald, J. R., and Claggett, O. T. (1953). Amyloid tumours of the lower part of the respiratory tract and mediastinum. Ann. Otol. (St. Louis), 62, 880.

Sorenson, G. D., and Shimamura, T. (1964). Experimental amyloidosis. III. Light and electron microscopic observations of renal glomeruli. Lab. Invest., 13, 1409.

Spiro, D. (1959). The structural basis of proteinuria in man. Electron microscopic studies of renal biopsy specimens from patients with lipid nephrosis, amyloidosis, and subacute and chronic glomerulonephritis. Amer. J. Path., 35, 47.

Symmers, W. St. C. (1956). Primary amyloidosis: A review. J. clin. Path., 9, 187.

Whitwell, F. (1953). Localised amyloid tumours of the lower respiratory tract. Thorax, 8, 309. 